\title{
Desarrollo sustentable y Medio ambiente
}

\author{
Sustainable development and environment \\ Diego Santos Durán ${ }^{a}$, Claudia Teresa Solano Pérez ${ }^{b}$
}

\begin{abstract}
:
The challenges of today's world imply a constant dynamic between competing and being the best in the shortest time and developing personally and professionally to achieve the best on the planet. However, the course of the planet has been wilting as much as nature; the days where trees could be enjoyed in environments without noise pollution are over, because wherever we go we hope to find electronic or battery operated devices that prevent us from fully enjoying the environment. We have thought that living nature will continue forever, but recent evidence shows day by day that the environment as we know it, is running out.
\end{abstract}

Keywords:

Environment, Economic and environmental development

\section{Resumen:}

Los desafíos del mundo actual implican una dinámica constante entre competir y ser el mejor en el menor tiempo y el desarrollarse personal y profesionalmente para conseguir lo mejor del planeta. Sin embargo, el curso del planeta se ha ido marchitando tanto como la naturaleza; los días donde los árboles se podían disfrutar en ambientes sin contaminación de ruido se acabaron, pues a cualquier lugar donde vayamos esperamos encontrar dispositivos electrónicos u operados con pilas y que nos impiden disfrutar completamente el medio ambiente. Hemos pensado que la naturaleza viva seguirá por siempre, pero las evidencias recientes demuestran día con día que el medio ambiente como lo conocemos, se está agotando.

Palabras Clave:

Medio ambiente, desarrollo económico y Ambiental.

\section{Introducción}

En este escrito se relatarán puntos clave para el desarrollo a futuro basado en la sustentabilidad y teniendo en cuenta al medio ambiente como principal mediador de nuestras acciones, así como un análisis del impacto que han tenido en nuestra demografía, el panorama actual y un posible futuro critico al que nos enfrentamos (1).

\section{Elementos iniciales sobre sustentabilidad y medio ambiente}

El término desarrollo sustentable ha tomado realce $\mathrm{e}$ importancia a partir de mediados del siglo XX encontrando su auge en la última década, volviéndose una realidad tangible las consecuencias del crecimiento demográfico desmedido y desorganizado al que ha llegado la humanidad en los últimos años, teniendo en cuenta la importancia del progreso socioeconómico se ha llegado al grado en el que no podemos continuar creciendo sin tener en cuenta el maltrato y desgaste a nuestros ecosistemas, siendo visible la sobreexplotación a la flora y fauna sin algún tipo de regulación durante un siglo entero, por lo que organismos internacionales se han visto forzados a intervenir de manera estricta en la conducta de la sociedad global entendiéndose así por decisión que "Desarrollo sustentable es el desarrollo que satisface las necesidades del presente sin comprometer la capacidad de las generaciones futuras para satisfacer sus propias necesidades". (3). Aunque pudiese

Pasante de Servicio Social, Universidad Autónoma del Estado de Hidalgo, Instituto de Ciencias de la Salud. Email: diegosd2705@gmail.com

${ }^{\mathrm{b}}$ Autor de Correspondencia. Universidad Autónoma del Estado de Hidalgo, Instituto de Ciencias de la Salud, https://orcid.org/0000-00034648-981X, Email: Claudia_solano@uaeh.edu.mx 


\section{Hidalgo, Vol. 8, No. 15 (2019) 68-70}

encontrarse sencilla esta definición la realidad de llevarla a cabo ha sido un reto casi insupurable para la humanidad, factores socioeconómicos como la industria y la explotación de la biosfera con fines turísticos e inclusive la simple satisfacción de la vida moderna y globalizada han hecho que el hombre deje una huella ecológica imborrable (El impacto de un individuo, ciudad o país, sobre la Tierra, para satisfacer lo que consume y para absorber sus residuos, se conoce como huella ecológica)

Para comprender mejor el desarrollo sustentable se deben tener conocimientos sobre los principios geofísicos que rigen a nuestro plantea, comprendiendo que somos materia y dependemos de materia se debe plantear la idea de que todos los recursos de nuestro planeta son finitos y que se encuentran sometidos a ciclos biogeofísicos autosustentables en perfecta armonía (1).

Ciclos biogeoquímicos: los compuestos químicos siguen distintas rutas en las que la materia pasa de su estado elemental a materia inorgánica, luego en orgánica para regresar de nuevo a su estado elemental.

Carbono: Es sólido a temperatura ambiente. Dependiendo de las condiciones de formación puede encontrarse en la naturaleza en distintas formas alotrópicas, carbono amorfo y cristalino en forma de grafito o diamante. Es el pilar básico de la química orgánica, y forma parte de todos los seres vivos.

Nitrógeno: Es un gas inerte, no metal, incoloro, inodoro e insípido que constituye aproximadamente las cuatro quintas partes del aire atmosférico, si bien no interviene en la combustión ni en la respiración.

Oxígeno: Es un gas a temperatura ambiente y representa aproximadamente el $20 \%$ de la composición de la atmósfera terrestre. Es uno de los elementos más importantes de la química orgánica; participa de forma muy importante en el ciclo energético de los seres vivos, esencial en la respiración celular de los organismos aeróbicos

Fósforo: Es un no metal que se encuentra en la naturaleza combinado en fosfatos inorgánicos y en organismos vivos, pero nunca en estado nativo. Es muy reactivo y se oxida espontáneamente en contacto con el oxígeno atmosférico emitiendo luz, dando nombre al fenómeno de la fosforescencia.

Azufre: Es un no metal abundante, insípido, inodoro. Se encuentra en sulfuros y sulfatos e incluso en forma nativa (especialmente en regiones volcánicas). Es un elemento químico esencial para todos los organismos y necesario para muchos aminoácidos y por consiguiente también para las proteínas. Se usa principalmente como fertilizante, pero también en la fabricación de pólvora, laxantes, cerillas e insecticidas.

Agua: Sustancia compuesta por un átomo de oxígeno y dos de hidrógeno. A temperatura ambiente es líquida, inodora, insípida e incolora. Se considera fundamental para la existencia de la vida.

Podemos entender así que la vida gira entorno a los elementos antes descritos, incluyendo por obviedad al hombre es por esto que se debe de priorizar la búsqueda de herramientas que le permitan a la sociedad seguir proliferando gradualmente sin que se vean comprometidos la biocenosis y el biotipo que componente a los ecosistemas de donde provienen nuestros recursos naturales. Por mencionar un ejemplo la energía eléctrica requerida para abastecer a nuestras grandes metrópolis es aun obtenida en su $70 \%$ a través combustibles fósiles, carbón mineral y gas LP, nuestra industria automotriz y transportista depende casi al 100\% del petróleo, recurso del cual es bien conocida su extrema dificultad de obtención (4).

Nuestra sociedad se basa en el consumo de recursos para mantener nuestro estilo de vida cómodo y globalizado en el que día a día se buscan nuevas formas de hacer nuestra existencia más placentera sin tener en cuenta las consecuencias catastróficas a nivel global que ya son una realidad el día de hoy y que sin duda serán peores si seguimos ignorando las señales que el planeta nos da en agonía.

Para concluir, es necesario expresar que el desarrollo sustentable no debe ser visto como una opción, si no como una obligación para nuestro crecimiento, como una nueva forma de vida y cultura a la cual debemos apegarnos, las fuentes de energía renovable deben ser nuestra mayor herramienta en esta nueva etapa de desarrollo social, así como la educación a las nuevas generaciones y el respeto por los ecosistemas y los nichos ecológicos de los cuales dependemos, podemos observar ya de manera tangible que las advertencias dadas en el Protocolo de Kioto no eran exageradas, cada día aumenta el número de incendios, huracanes y demás desastres naturales, esto no cambiara su curso mientras la industria y la posición socioeconómica sigan siendo de mayor importancia que nuestros bosques, selvas y mares.

El planeta sigue su curso y para él, el tiempo es efímero e infinito, la vida en él puede surgir y extinguirse todos los días por el resto de la eternidad, entonces dime tu 
Publicación semestral, Educación y Salud Boletín Científico Instituto de Ciencias de la Salud Universidad Autónoma del Estado de

Hidalgo, Vol. 8, No. 15 (2019) 68-70

que no eres eterno ¿Cuánto tiempo más quieres de este planeta para ti?

\section{Referencias}

[1] Pérez-García, M. y Hernández-Cárdenas, G. (1998) Desarrollo sustentable y globalización. Rev Electrónica de Cultura Científica. UNAM, Facultad de Ciencias. México.

[2] Vilches, A., Gil-Pérez, D., Toscano, J.C, Macías, O. (2014) Rev. Economía y sostenibilidad. Programa de Acción Social. OEI. España.
[3] Varea, A., Vargas, MT., Barrezueta, L., López-Parodi, J. (1995) Bosques, Árboles y Comunidades Rurales - Fase II - Documento de Trabajo: La Radio y Procesos Participativos de Desarrollo Sostenible en la Región Amazónica. ONU, FAO. Chile.

[4] Ramírez Treviño, Alfredo; Sánchez Núñez, Juan Manuel; García Camacho, Alejandro. (2004) El Desarrollo Sustentable: Interpretación y Análisis. Rev del Centro de Investigación. Universidad La Salle, vol. 6, núm. 21. México. 